\title{
Angermüller, Johannes; Nonhoff, Martin; Herschinger, Eva; Macgilchrist, Felicitas; Reisigl, Martin; Wedl, Juliette; Wrana, Daniel; Ziem, Alexander (Hrsg.) (2014): Diskursforschung. Ein interdisziplinäres Handbuch.
}

\author{
Bielefeld: Transcript. 2 Bände im Schuber, kart., zahlr. Abb., 1259 S.
}

\section{Boris Michel ${ }^{1}$}

Online publiziert: 22. Juni 2016

(c) Springer-Verlag Berlin Heidelberg 2016

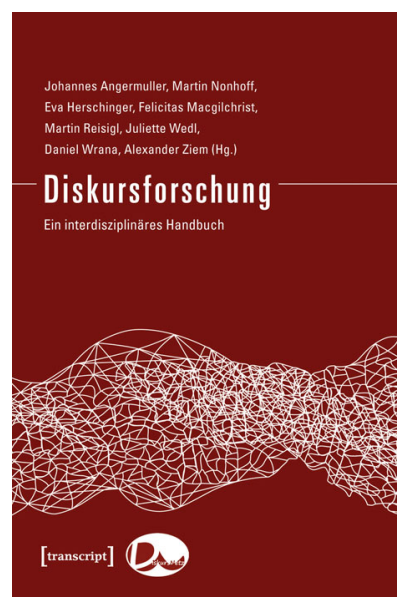

Diskursforschung hat sich in den deutschsprachigen Sozial-, Kultur- und Geisteswissenschaften in den letzten Jahren zu einem ausgesprochen lebhaften und vielfältigen Forschungsfeld entwickelt. So liegt es nahe, den Versuch zu unternehmen, dieses Feld in einem dezidiert ,,interdisziplinären Handbuch“ zu bündeln und die vielen Stränge zwischen zwei (bzw. in diesem Falle vier) Buchdeckeln zu versammeln. Herausgekommen ist dabei ein Handbuch, das aufgrund seiner Anlage und der Vielfältigkeit des Forschungsfeldes allerdings nicht sonderlich handlich ist. In zwei dicken Bänden, in über 60 Kapiteln von 50 Autorinnen und Autoren und auf über 1200 Seiten bieten die

Dr. Boris Michel

boris.michel@fau.de

1 Institut für Geographie, Friedrich-Alexander-Universität Erlangen-Nürnberg, Wetterkreuz 15, 91058 Erlangen, Deutschland im Rahmen des „DiskursNetz“" entstandenen Texte einen breiten Überblick über Felder, Theorien und Methoden der Diskursforschung. Damit wird in erster Linie ein weiterer Beitrag zum wachsenden Feld der Überblicks- und Einführungsbücher zur Diskursforschung geleistet.

Band $1 \mathrm{zu}$,Theorien, Methodologien und Kontroversen“ enthält neben der Einleitung drei Teile: einen Teil über ,Disziplinäre und transdisziplinäre Felder der Diskursforschung“, einen Teil über „Konzepte und Kontroversen der Diskurstheorie“ und einen Teil zu „Grundfragen der Forschungspraxis. Epistemologie, Methodologie und Forschungsdesign“ (der selbst noch einmal aus drei Unterteilen besteht). Band 2 ist ausgerichtet auf ,Methoden und Analysepraxis“ der Diskursforschung und präsentiert gut 20 exemplarische Arbeiten, die sich aus unterschiedlichen Perspektiven der Diskursforschung mit Hochschulreformdiskursen befassen. Hieran wird die methodische Vielfältigkeit der Diskursforschung deutlich. In den meisten Fällen sind die Autorinnen und Autoren in beiden Bänden vertreten, sodass grundsätzlich eine gute Verzahnung und gegenseitige Bezugnahme der Beiträge hergestellt wird.

Einem solch umfassend ansetzenden Werk im Rahmen einer kurzen Besprechung gerecht $\mathrm{zu}$ werden ist schwerlich möglich, zumal es gerade die Heterogenität und Reflexivität ist, die das Buch auszeichnet und worin sowohl seine Stärke wie auch sein größtes Problem liegen. Wenngleich es in seinem Auftreten Kanonbildung zu beanspruchen scheint und stark diskurspolitisch agiert, so versuchen die Herausgeber und Herausgeberinnen das Feld und die Begriffe offenzuhalten. So wird beispielsweise kein verbindender Diskursbegriff den Bänden vorangestellt, sondern eine Reihe von Merkmalen angeführt, welche „,von vielen

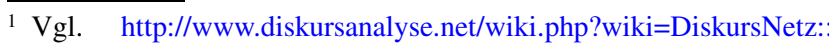
DiskursNetz.
} 
DiskursforscherInnen geteilt werden“ (Band 1: $18 \mathrm{f}$.). Dazu wird etwa die Vorstellung angeführt, dass Diskurs konstitutiv sei für das Soziale, dass Diskurs situierte Praxis sei, dass Diskursforschende sich meist für „Probleme mit einer gewissen gesellschaftlichen Relevanz" (Band 1: 19) interessieren, dass gegen akteurszentrierte Ansätze eine diskursive Konstruktion von Subjektivität angenommen wird und dass Diskurse eine Materialität haben. Hiernach beschäftigt sich die Einleitung des Bandes in erster Linie damit aufzuzeigen, wie vielschichtig die Verortung von Diskursforschung ist, wie vielfältig die Inspirationen und die Ausformungen von Diskurstheorien und Diskursanalysen sind und welche Schwerpunkte, Differenzen und welche ,,nationalen 'Schulen“ (Band 1: 27) sich im Vergleich der Rezeptionen unter anderem im englisch-, französisch-, spanisch- und deutschsprachigen Raum ausgebildet haben. Diese Betonung der Differenz, Vielfalt und Offenheit zieht sich auch durch die nachfolgenden Beiträge.

In Band 1, Teil 1 (S. 39 ff.) werden zunächst disziplinäre Differenzen in den Mittelpunkt gerückt. In 16 Kapiteln werden Geschichte und Figuration von Diskursforschung in so unterschiedlichen Disziplinen wie beispielsweise Philosophie, Linguistik, Soziologie, Gender Studies und auch Humangeographie skizziert. Deutlich wird dabei die sehr unterschiedliche Einbindung von Diskursforschung in verschiedene disziplinäre Denk- und Arbeitsweisen. Dass es dabei zu Redundanzen kommt und disziplininterne Besonderheiten etwas opak bleiben, ist wenig überraschend und in dieser Form vermutlich nicht zu vermeiden; es mindert aber gleichwohl das Lesevergnügen.

Teil 2 (S. 343 ff.) diskutiert in sechs Kapiteln zentrale Konfliktlinien und Konzepte der Diskursforschung - darunter auch thematische ,Klassiker" wie Subjekt, Macht oder Materialität. $\mathrm{Ob}$ die in diesen Kapiteln teilweise gewählte Form fiktiver Dialoge und Szenen, die einmal mehr die Offenheit deutlich machen soll, nun zugleich auch die ,intellektuelle Fantasie" befeuert, wie es die thematische Einleitung verspricht (Band 1:343), mag unterschiedlich bewertet werden. Der Rezensent hatte eher den Eindruck, dass hier etwas unglücklich zwischen Oberflächlichkeit und InsiderJargon operiert wurde und so weder eine Einführung noch eine Vertiefung so recht gelungen ist.

Der dritte Teil des ersten Bandes (S. 479 ff.) widmet sich den „Grundfragen der Forschungspraxis“ unter den Stichworten „Epistemologie, Methodologie und Forschungsdesign“. Diskutiert werden dabei zunächst Fragen nach der Übersetzung des Diskursbegriffs in eine Methode der empirischen Sozialwissenschaften. Dass diese Methodisierung dem Ethos Foucaults, der als ,Säulenheiliger' durch die Beiträge wandelt, möglicherweise zuwiderlaufen würde, diskutiert das lesenswerte Gespräch zwischen Robert Feustel, Reiner Keller, Dominik Schrage, Juliette Wedl, Daniel Wrana und ihrer Moderatorin Silke van Dyk (S. 482 ff.).
Hieran anschließend werden stärker methodologische bzw. forschungspraktische Fragen diskutiert, hier bezeichnet als „Knotenpunkte des Forschungsprozesses“ (Band 1: 614). Zudem werden Schritte über die Grenzen der Diskursforschung hinaus in Richtung auf andere Forschungsperspektiven (unter anderem Hermeneutik, Inhaltsanalyse, linguistische Pragmatik) unternommen.

Der zweite Band, der mit dem vierten Teil zusammenfällt, greift auf über 500 Seiten das Thema „Hochschulreform" exemplarisch heraus und illustriert daran in „kaleidoskopartigem Blick“ (Band 2: 15) unterschiedliche methodische Zugänge der Diskursforschung. Die Idee ist überzeugend, die Realisierung aber leider nur eingeschränkt gelungen. Die Anwendungen sind nur relativ lose mit dem konzeptionellen Teil verbunden und es werden auch hier eigenständige Einführungen in die jeweiligen Methoden diskutiert. Dies führt letztlich dazu, dass die meisten der hier versammelten Beiträge auf einigen Seiten in die Methode einführen und auf einigen Seiten diese Methode durchexerzieren. Dabei kommt es kaum zu einer Diskussion der Bezüge zwischen den Methoden, ebenso wenig werden im Ganzen gesehen mehr als oberflächlich bleibende Einführungen erreicht.

Insgesamt scheitert das Projekt an seinem uneinlösbaren Anspruch, ,interdisziplinäre Arbeitszusammenhänge“ zu schaffen, ,in denen disziplinäre Tendenzen nicht additiv nebeneinander“ gestellt werden, sondern ,inhaltlich verschränkt" sind (Band 1: 13). Dabei ist das Buch gut strukturiert und es wurde angesichts der Breite an Beiträgen versucht, der Gefahr des Auseinanderfallens durch eine klare und gut moderierte Form zu begegnen. Dem Handbuch ist anzusehen, dass es weit mehr ist als ein weiterer Sammelband zum Thema, sondern dass es das Ergebnis einer langen und intensiven Diskussion eines größeren Arbeitszusammenhanges darstellt. Aber möglicherweise liegt auch darin das Problem. Wenn Diskursforschung als eine Perspektive „am Schnittpunkt von Sprache und Gesellschaft“ (Band 1: 13) verstanden wird, für die es konstitutiv ist, dass sie offen und in erheblichem Maße undiszipliniert ist und eine strenge Methodisierung von den Autorinnen und Autoren aus gutem Grund abgelehnt wird, so droht die Betonung von Heterogenität und der internen Differenzen von Diskursforschung in diesem Band in Indifferenz umzuschlagen. Es bleibt ein Nebeneinander, das aber nicht so recht produktiv wird.

Das verweist schließlich auf eine Unklarheit bezüglich des Zielpublikums. Als Einführung in Diskursforschung ist das Werk zu speziell und aufgrund seines Umfangs wenig zugänglich. Hier sind sicherlich die zahlreichen speziellen Einführungen hilfreicher, die jeweils in sich konsistente Diskursbegriffe entwickeln, diese von anderen abgrenzen und aufzeigen, wie damit relevante Ergebnisse zu relevanten Themen hervorgebracht werden können. Andererseits 
sind die Beiträge in den vorliegenden Bänden dann oftmals doch zu einführend oder bleiben zu oberflächlich, um neue Forschungsfragen vertieft zu bearbeiten.

Das bedeutet gewiss nicht, dass nicht vieles lesenswert, spannend und erhellend ist und so Wichtiges zum Feld der Diskursforschung beigetragen wird. Für Forschung und
Lehre ist hier so manches nützlich. Aber als ein interdisziplinäres Handbuch, das beansprucht, dieses Feld der Diskursforschung zu prägen, das Diskursforschung als kritische Perspektive auf gesellschaftliche Problematisierungen attraktiv machen soll, funktioniert dieses Werk nur eingeschränkt. 